\title{
Imaging a Phase Change in Kagome Spin Ice
}

\author{
Polarized neutron scattering images of holmium titanate show how a \\ kagome ice changes topology in a magnetic field.
}

By Marric Stephens

$\mu$ spin ice is a "frustrated" magnetic material whose spins are arranged in a nontrivial geometric configuration.

Now, researchers have performed a comprehensive numerical and experimental study of a "kagome" ice, which is similar to a spin ice but forms in the presence of a magnetic field [1]. They show that the spin topology of a kagome ice can be tuned by varying the orientation of that field.

Alexandra Turrini at the Paul Scherrer Institute, Switzerland, and colleagues studied holmium titanate, in which magnetic holmium ions form interconnected tetrahedrons. In the absence of a magnetic field, holmium titanate is a 3D spin ice, with the spins at two vertices of each tetrahedron pointing inward and the other two pointing outward. Applying a magnetic field along the [111] direction of the material fixes the spin of the holmium ion aligned with the field, leaving the remaining three to define the kagome lattice. This $2 \mathrm{D}$ lattice is composed of the tetrahedrons' triangular bases, with two spins pointing outward and one spin pointing inward, or vice versa.

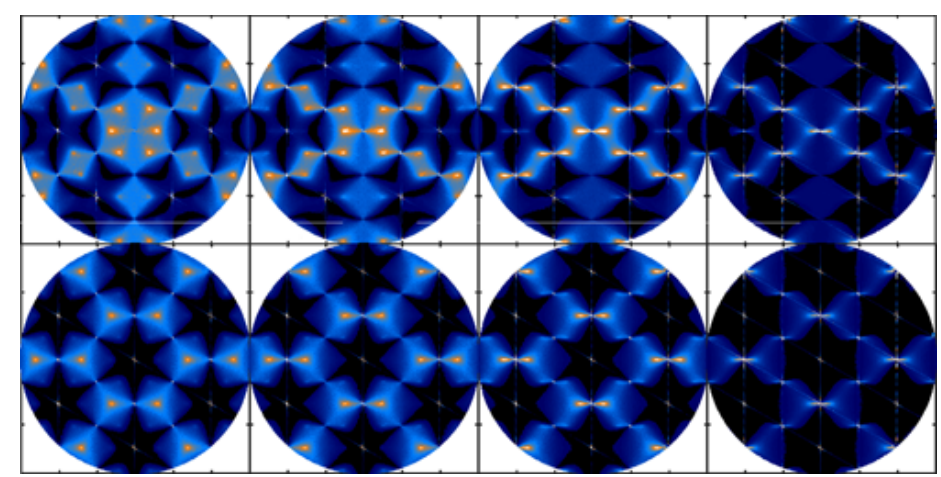

Credit: A. A. Turrini et al. [1]
Turrini and colleagues used polarized neutron scattering to analyze the spin directions of the holmium ions in their kagome ice as they varied the orientation of the magnetic field. In agreement with their numerical simulations, they observe features that indicate that as the field deviates from the [111] direction, the material's spin topology undergoes an unconventional phase transition. The precise nature of this transition depends on the temperature and the magnitude and direction of the field. The researchers say that their experiment offers an unusual example of a precisely controllable topological phase change. It also provides an experimental benchmark for future spin-ice studies.

Marric Stephens is a Corresponding Editor for Physics based in Bristol, UK.

\section{REFERENCES}

1. A. A. Turrini et al., "Tunable critical correlations in kagome ice," Phys. Rev. B 105, 094403 (2022). 